\title{
GROUP RINGS IN WHICH EVERY LEFT IDEAL IS A RIGHT IDEAL
}

\author{
P. MENAL
}

\begin{abstract}
Let $K[G]$ denote the group ring of $G$ over the field $K$. In this note we characterize those group rings in which all left ideals are right ideals.
\end{abstract}

Let $R$ be a ring. We say that $R$ is l.i.r.i. if every left ideal is a right ideal. A ring is l.a.r.i. if every left annihilator is a right ideal. Our notation follows that of [2].

The main results are

Theorem I. Let $K$ be a field and let $G$ be a nonabelian periodic group. Then if $K[G]$ is l.a.r.i. one of the following occurs

(i) Char $K=0$ and $G$ is a Hamiltonian group such that for each odd exponent, $n$, of $G$ the quaternion algebra over the field $K\left(\xi_{n}\right)$, where $\xi_{n}$ is a primitive nth root of unity, is a division ring.

(ii) Char $K=2$ and $K$ does not contain any primitive cube root of unity. Moreover $G \simeq Q \times A$, where $Q$ is the quaternion group of order 8 and $A$ is abelian in which each element has odd order and if $n$ is an exponent for $A$, the least integer $m>1$ satisfying $2^{m} \equiv 1(\bmod n)$ is odd.

Conversely if $K[G]$ satisfies either (i) or (ii), then $K[G]$ is l.i.r.i. and, in particular, it is l.a.r.i.

Observe that if char $K>2$ and $G$ is periodic, then $K[G]$ is 1.a.r.i. if and only if $G$ is abelian.

THEOREM II. Let $K[G]$ denote the group ring over a nonabelian group. Then the following are equivalent

(i) $K[G]$ is l.i.r.i.

(ii) $G$ is locally finite and if $\alpha, \beta \in K[G]$ with $\alpha \beta=0$, then $\beta \alpha=0$.

(iii) $G$ is locally finite and $K[G]$ is l.a.r.i.

If we combine the above theorems we get necessary and sufficient conditions for $K[G]$ to be l.i.r.i.

By using the antiautomorphism of $K[G]$ given by $\Sigma_{x \in G} k_{x} x \mapsto$ $\sum_{x \in G} k_{x} x^{-1}$ we see that $K[G]$ is l.i.r.i. (1.a.r.i.) if and only if $K[G]$ is r.i.l.i. (r.a.l.i.).

Received by the editors February 2, 1978.

AMS (MOS) subject classifications (1970). Primary 16A26.

Key words and phrases. Group ring, quaternion algebra, Hamiltonian group.

(c) 1979 American Mathematical Society 0002-9939/79/0000-0403/\$02.25 
LEMMA 1. (i) $K[G]$ is l.i.r.i. if and only if for every finitely generated subgroup $H \subseteq G, K[H]$ is l.i.r.i. (ii) If $K[G]$ is l.i.r.i., then all subgroups of $G$ are normal. (iii) Suppose that $G$ is periodic. If $K[G]$ is l.a.r.i., then all subgroups of $G$ are normal.

Proof. (i) First we suppose that for every finitely generated subgroup $H \subseteq G, K[H]$ is l.i.r.i. Let $I \subseteq K[G]$ be a left ideal. Let $\alpha \in I, g \in G$. We set $H=\langle g$, Supp $\alpha\rangle$. Then $I \cap K[H]$ is a left ideal of $K[H]$ and hence $I \cap K[H]$ is an ideal of $K[H]$, since $H$ is finitely generated. Now $g \in H$ and $\alpha \in I \cap K[H]$ so $\alpha g \in I \cap K[H] \subseteq I$. Therefore we have shown that $I g \subseteq$ $I$ for any $g \in G$ and so $I$ is a right ideal. Conversely let $H$ be a subgroup of $G$ and suppose that $I \subseteq K[H]$ is a left ideal of $K[H]$. Let $\left\{x_{i}\right\}$ be a set of left coset representatives for $H$ in $G$. Then $K[G]$ is a free right $K[H]$-module with basis $\left\{x_{i}\right\}$. Thus we have $K[G]=\dot{\Sigma} x_{i} K[H]$. Denote $\dot{\Sigma} x_{i} I$ by $J$. Clearly $J$ is a left ideal of $K[G]$. If we suppose that $K[G]$ is l.i.r.i., then we have that $J$ is a right ideal of $K[G]$. Let $h \in H$. Then

$$
I h \subseteq J h \cap K[H] \subseteq J \cap K[H]=I
$$

and so $I$ is a right ideal of $K[H]$.

(ii) In order to prove that all subgroups of $G$ are normal it suffices to see that all cyclic subgroups are normal. Let $a, g \in G$. Consider the left ideal $I=K[G](1-a)$. Then $I$ is an ideal, since $K[G]$ is l.i.r.i. Thus $g^{-1}(1-a) g$ $\in I$ and $1-g^{-1} a g=\alpha(1-a)$ for a suitable element $\alpha \in K[G]$. Now we use the $K[\langle a\rangle]$-homomorphism $\theta: K[G] \rightarrow K[G]$ in which $\sum_{x \in G} k_{x} x \mapsto$ $\Sigma_{x \in\langle a\rangle} k_{x} x$ and we obtain $1-\theta\left(g^{-1} a g\right)=\theta(\alpha)(1-a)$. Since $1-a$ is not invertible we have that $\theta\left(g^{-1} a g\right) \neq 0$. Hence $g^{-1}$ ag $\in\langle a\rangle$.

(iii) Suppose that $G$ is periodic and $K[G]$ is l.a.r.i. Let $g \in G$. Set $H=\langle g\rangle$. Lemma 1.2 [2, Chapter 3] yields that $l(\hat{H})=K[G] \omega(K[H])$. On the other hand we have that $H=\{x \in G: x-1 \in K[G] \omega(K[H])\}$. By hypothesis $l(\hat{H})$ is an ideal, then it is easy to see that $H$ is normal in $G$.

We recall that a nonabelian group $G$ such that all subgroups are normal is a Hamiltonian group, that is [ $\mathbf{1}$, Theorem 12.5.4]

$$
G=Q \times A \times B
$$

where $Q$ is the quaternion group of 8 elements, $A$ is an abelian group such that every element has odd order, and $B$ is an abelian group of exponent 2 . For the rest of this paper we fix this notation.

LEMMA 2. Suppose that $K[G]$ is l.a.r.i. Let $\alpha, \beta \in K[G]$ such that $\alpha \beta=0$. Then $\beta \alpha=0$.

Proof. Suppose $I$ and $J$ are ideals of $K[G]$ with $I J=0$. Then $J I$ is an ideal of $K[G]$ and $\operatorname{tr}(J I)=\operatorname{tr}(I J)=0$. Thus $J I=0$ since any ideal of trace zero is zero. Now let $K[G]$ be 1.a.r.i. If $\alpha, \beta \in K[G]$ with $\alpha \beta=0$, then $I J=0$ where $I=K[G] \alpha K[G]$ and $J=K[G] \beta K[G]$. Thus $J I=0$ and hence $\beta \alpha=0$.

In characteristic 2 we need the following 
LemMa 3. Let $K$ be a field of characteristic 2. Suppose that $K$ does not contain any primitive cube root of unity. Put

$$
Q=\left\langle a, b \mid a^{2}=b^{2}, a^{4}=1, b^{-1} a b=a^{-1}\right\rangle .
$$

Then if $\alpha=\Sigma k_{x} x \in K[\langle a\rangle]$ such that $|\alpha|=1$ (where $|\alpha|=\Sigma k_{x}$ ) we have

$$
1+(\alpha b)^{2}=\left(1+a^{2}\right) u
$$

where $u \in K[\langle a\rangle]$ is a unit.

Proof. Let $\alpha=k_{1}+k_{2} a+k_{3} a^{2}+k_{4} a^{3} \in K[\langle a\rangle]$ with $\Sigma k_{i}=1$. Then a calculation proves that

$$
1+(\alpha b)^{2}=\left(1+a^{2}\right)\left(1+\left(k_{1}+k_{3}\right)\left(k_{2}+k_{4}\right) a\right) .
$$

Since $Q$ is a 2-group and char $K=2$ we know that $K[Q]$ is a local ring whose maximal ideal is $\{\alpha \in K[Q]:|\alpha|=0\}$. Suppose by contradiction that $1+\left(k_{1}\right.$ $\left.+k_{3}\right)\left(k_{2}+k_{4}\right) a$ is not a unit. Then $\left(k_{1}+k_{3}\right)\left(k_{2}+k_{4}\right)=1$, and since $\Sigma k_{i}=$ 1 we see that $k_{1}+k_{3}$ is a primitive cube root of unity. Since $K$ does not contain any primitive cube root of unity we have a contradiction.

The PROOF of TheOrem I. Suppose that $G$ is a nonabelian periodic group and $K[G]$ is 1.a.r.i. Then Lemma 1 (iii) yields that $G=Q \times A \times B$. First we observe that the case char $K>2$ is not possible. Since $K[G]$ is 1.a.r.i. clearly $K[Q]$ so is. But in char $>2$ we have

$$
K[Q] \cong K+K \dot{+} K \dot{+} K \dot{+} M(2, K)
$$

and this is a contradiction, since $M(2, K)$ is not 1.a.r.i. Suppose char $K=0$. Let $n$ be an exponent for $A$ and let $x \in A$ such that $o(x)=n$. Then $K[\langle x\rangle]$ is a direct sum of fields

$$
K[\langle x\rangle] \cong K\left(\xi_{n}\right)+L_{1} \dot{+} \cdot \dot{+} L_{m}
$$

where $o\left(\xi_{n}\right)=n$. On the other hand we have

$$
K[Q] \simeq K \dot{+} K \dot{+} K \dot{+} K \dot{+}((-1,-1) / K)
$$

where the last summand is the quaternion algebra over $K$. Since $K[Q \times\langle x\rangle]$ $\simeq K[Q] \otimes_{K} K[\langle x\rangle]$ we get that $((-1,-1) / K) \otimes K\left(\xi_{n}\right) \simeq\left((-1,-1) / K\left(\xi_{n}\right)\right)$ is a direct summand of $K[Q \times\langle x\rangle]$ and so $\left((-1,-1) / K\left(\xi_{n}\right)\right)$ is 1.a.r.i. Therefore the quaternion algebra over $K\left(\xi_{n}\right)$ is a division ring. Conversely suppose that $K[G]$ satisfies (i). Then we will prove that $K[G]$ is 1.i.r.i. It follows from Lemma $1(i)$ that it suffices to consider $G$ finite. Then

$$
G \cong Q \times A \times(Z / 2 Z) \times \cdots \times(Z / 2 Z)
$$

( $m$ copies of $Z / 2 Z$ ) and we get

$$
K[G] \cong K[Q \times A]+\cdots+K[Q \times A]
$$

( $2^{m}$ copies of $\left.K[Q \times A]\right)$. Clearly we can suppose that $G=Q \times A$. Then it is easy to see that

$$
K[G] \cong K[A]+K[A]+K[A]+K[A]+\prod_{i}\left(\frac{-1,-1}{K\left(\xi_{i}\right)}\right)
$$


where $o\left(\xi_{i}\right)$ are exponents for $A$. Hence we see that $K[G]$ is a product of 1.i.r.i. rings. Therefore $K[G]$ is l.i.r.i.

Char $K=2$. First we observe that if $K$ contains a primitive cube root of unity then $K[G]$ is not 1.a.r.i. From Lemma 2 it suffices to exhibit elements $\alpha, \beta \in K[G]$ such that $\alpha \beta=0$ but $\beta \alpha \neq 0$. If $\xi$ is a primitive cube root of unity we set

$$
\begin{aligned}
& \alpha=(1+\xi(1+\xi a) b), \\
& \beta=(1+\xi(1+\xi a) b)(1+a) b .
\end{aligned}
$$

A calculation proves that $\alpha \beta=0$ but $\beta \alpha \neq 0$. We now prove that $G=Q \times$ $A$. If this is not the case there exists an element $x \in G-Q$ of order 2 which centralizes $G$. Again there exist elements

$$
\begin{aligned}
& \alpha=1+(a+b+a b) x, \\
& \beta=(a+b+a b)(1+a)+(1+a) x,
\end{aligned}
$$

such that $\alpha \beta=0$ but $\beta \alpha \neq 0$ and so $K[G]$ is not l.a.r.i. Let $n$ be an exponent for $A$ and $x \in A$ such that $o(x)=n$. Since char $K=2$ we have that $K[\langle x\rangle]$ is semisimple, and so

$$
K[\langle x\rangle]=K\left(\xi_{n}\right)+\cdots+L_{m} \text { where } o\left(\xi_{n}\right)=n .
$$

Then $K[Q] \otimes K\left(\xi_{n}\right) \cong K\left(\xi_{n}\right)[Q]$ is a direct factor of $K[Q \times\langle x\rangle]$. By hypothesis $K\left(\xi_{n}\right)[Q]$ is 1.a.r.i. By above $K\left(\xi_{n}\right)$ does not contain any primitive cube root of unity. Therefore $24 m$, where $m$ is the degree of the extension $\left(Z / 2 Z\left(\xi_{n}\right)\right) /(Z / 2 Z)$. But $m$ is precisely the least integer satisfying $2^{m} \equiv 1$ $(\bmod n)$. Conversely suppose that $K[G]$ satisfies' (ii). We shall prove that $K[G]$ is 1.i.r.i. Again from Lemma 1(i) we can consider that $G$ is finite. Then

$$
K[A] \cong K\left(\xi_{i}\right) \dot{+} \cdots \dot{+} K\left(\xi_{m}\right)
$$

and so

$$
K[Q \times A] \cong K\left(\xi_{1}\right)[Q] \dot{+} \cdots \dot{+} K\left(\xi_{m}\right)[Q] .
$$

By hypothesis the field $K\left(\xi_{i}\right)$ does not contain any primitive cube root of unity. Since a product of 1.i.r.i. rings is 1.i.r.i., we have only to prove that if a field $K$ does not contain any primitive cube root of unity, then $K[Q]$ is 1.i.r.i. Let $I \subseteq K[G]$ be a left ideal. Suppose that $\alpha \in I$. We can write $\alpha$ in the form $\alpha=\alpha_{1}+\alpha_{2} b$, where $\alpha_{i} \in K[\langle a\rangle]$. The first task is to show that $\alpha_{i}\left(1+a^{2}\right) \in$ $I$. Note that if $\alpha_{1}\left(1+a^{2}\right) \in I$, then, since $1+a^{2}$ is central, $\alpha_{2} b\left(1+a^{2}\right) \in I$. Again $\alpha_{2}\left(1+a^{2}\right)$ is central and therefore $b \alpha_{2}\left(1+a^{2}\right) \in I$. Since $I$ is a left ideal $\alpha_{2}\left(1+a^{2}\right) \in I$. Thus we need only to prove that $\alpha_{1}\left(1+a^{2}\right) \in I$. If $\alpha$ is a unit, then $I=K[Q]$. Thus we may suppose that $\alpha$ is not a unit. Then we have $\left|\alpha_{1}\right|+\left|\alpha_{2}\right|=0$. Suppose that $\alpha_{1}$ is a unit. Then $1+\alpha_{1}^{-1} \alpha_{2} b \in I$. Clearly $1+\left(\alpha_{1}^{-1} \alpha_{2} b\right)^{2} \in I$, so Lemma 3 yields that $1+a^{2} \in I$. Hence $\alpha_{1}\left(1+a^{2}\right) \in$ I. If $\alpha_{1}$ is not a unit, then we have $\left|\alpha_{1}\right|=0$ and hence $\left|\alpha_{2}\right|=0$. Therefore $\alpha_{1}=\beta_{1}(1+a)$ and $\alpha_{2}=\beta_{2}(1+a)$ for suitable elements $\beta_{i} \in K[\langle a\rangle]$. Thus $\alpha=\left(\beta_{1}+\beta_{2} a b\right)(1+a)$. If $\beta_{1}+\beta_{2} a b$ is a unit we obtain that $1+a \in I$ and 
so $\alpha_{1}\left(1+a^{2}\right)=\alpha_{1}(1+a)^{2} \in I$. Hence we may consider that $\left|\beta_{1}\right|+\left|\beta_{2}\right|=$ 0. If $\beta_{1}$ is a unit, then $\left(1+\beta_{1}^{-1} \beta_{2} a b\right)(1+a) \in I$. Again we use Lemma 3 and we get that $\left(1+a^{2}\right)(1+a) \in I$. Thus $\alpha_{1}\left(1+a^{2}\right)=\beta_{1}(1+a)\left(1+a^{2}\right) \in I$. Finally if $\beta_{1}$ is not a unit we have $\beta_{1}=\gamma_{1}(1+a)$ for certain $\gamma_{1} \in K[\langle a\rangle]$. Therefore $\alpha_{1}\left(1+a^{2}\right)=\gamma_{1}\left(1+a^{2}\right)\left(1+a^{2}\right)=0$ and, certainly, $\alpha_{1}\left(1+a^{2}\right) \in$ $I$. Now we will prove that $\alpha x \in I$ for any $x \in Q$. Since $Q=\langle a, b\rangle$ it suffices to see that $\alpha a, \alpha b \in I$. By using the automorphism of $Q$ given by $a \mapsto b$, $b \mapsto a$ we see that we have only to prove that $\alpha a \in I . \alpha_{2}\left(1+a^{2}\right)$ is central and so

$$
\alpha a=\alpha_{1} a+\alpha_{2} b a=a \alpha+a b \alpha_{2}\left(1+a^{2}\right) .
$$

Since $a \alpha \in I$ and by above $\alpha_{2}\left(1+a^{2}\right) \in I$, the result follows.

THE PROOF OF THEOREM II. (i) $\Rightarrow$ (ii). It follows from Lemma 1(ii) that all subgroups of $G$ are normal. Since $G$ is not abelian, it is a Hamiltonian group and, clearly, locally finite. Since if a ring is 1.i.r.i., then it is 1.a.r.i. Lemma 2 completes the proof. Trivially (ii) implies (iii). It follows from Theorem I that (iii) implies (i). The result follows.

\section{REFERENCES}

1. M. Hall, Jr., The theory of groups, Macmillan, New York, 1959.

2. D. S. Passman, The algebraic structure of group rings, Wiley-Interscience, New York, 1977.

Universitat Autònoma, Secció de Matemàtiques, Bellaterra, Barcelona, Espanya 